\title{
Evaluation of PCR-based methods and ribotyping performed with a mixture of Pst I and SphI to differentiate strains of Salmonella serotype Enteritidis
}

\author{
ELENA LANDERAS and M. CARMEN MENDOZA
}

Área de Microbiología, Departamento de Biología Funcional, Universidad de Oviedo, 33006 Oviedo, Spain

\begin{abstract}
The capacity to differentiate Salmonella serotype Enteritidis strains by PCR ribotyping; RAPD typing with three arbitrary primers and ribotyping with a mixture of Pst I and $S p h$ I or 'PS ribotyping', was evaluated on a series of 65 strains associated with human infections and 11 reference strains. The series had been analysed previously by phage typing and ribotyping performed with $P s t I$ and $S p h$ I, separately. All methods typed all the strains; however, only ribotyping showed good reproducibility and sensitivity. Twenty-two PS ribotypes (discrimination index $=0.74$ ) were identified, differentiating strains ascribed to seven phage types (PTs 1, 4, 6, 6a, 7, 8 and RDNC) as well as phage untyped strains. Conversely, some strains of PTs, 1, 4, 5a, 6, 6a, 7, 34 and RDNC showed the most frequent PS ribotype. By PCR ribotyping a single profile was found; while by RAPD typing, one, two or three RAPD types were identified with the primers MK22, OPB6 and OPB17, respectively. All Spanish strains were assigned to a single combined RAPD type, except PT11 strains which showed a different and specific RAPD type with OPB17. The banding patterns defining the PS ribotypes were interpreted more easily and the patterns could be compared more accurately than the banding patterns defining RAPD types. A similarity dendrogram generated from the 22 PS ribotypes was traced and compared with RAPD types and phage types. Data from this work indicated that 'PS ribotyping' was the most useful genetic procedure to differentiate Enteritidis strains, and, therefore, it can be used as a complementary or alternative typing method to phage typing within this serotype.
\end{abstract}

\section{Introduction}

From the early 1980s Salmonella serotype Enteritidis has emerged as the serotype most commonly associated with human salmonellosis in many European countries and similar findings have been reported in the USA in the 1990 s [1-6]. It is a zoonotic serotype whose main reservoir is poultry $[7,8]$. Nowadays, improved epidemiological surveillance is necessary because of the continuing presence of disease in both industrial and developing countries, and the rapidly growing international foods trade between countries - with different endemic and epidemic lineages of Enteritidis and maintaining different levels of hygiene in the production and manufacture of both human foods and animal

Received 17 June 1997; revised version accepted 6 Sept. 1997.

Corresponding author: Professor M. C. Mendoza. feeds $[2,9]$. Provided that typing antisera are available, the identification of strains as Enteritidis rarely causes problems in microbiology laboratories. However, to obtain a better understanding of the public health hazard caused by different types or clones, the further differentiation of Enteritidis strains will usually be required to ascertain the circulation, endemic level, incidence, prevalence and evolution of pathogenic clones through space and time. It also enables one clinical case to be associated with other sporadic or outbreak cases, reservoirs and transmission vehicles. The success of these aims depends on methods that distinguish strains from different sources, and various techniques, phenotypic as well as genotypic, have been reported as useful. Among the former, phage typing plays a central role in epidemiological studies, the scheme of Ward et al. [4] being the most frequently used. There is now no doubt that a few phage types in particular PT4 in Europe and PT8 and PT13a in the USA - are most frequently associated with food- 
poisoning and with the concurrent widespread infection of commercial poultry meat and egg dishes $[2,3,7,10-$ 13].

An important effort to develop an accurate genotypic typing method for Enteritidis is being made and several techniques have been evaluated over the present decade $[3,5,6,8,10,11,13,14]$. The most recently introduced assays for DNA fingerprinting are the PCR-based typing methods. Among these is PCR ribotyping, which analyses the spacer regions of $r r n$ operons with oligonucleotide primers complementary to the conserved regions of the $16 \mathrm{~S}$ and $23 \mathrm{~S}$ rRNA genes [15]. Other methods are based on the random amplification of polymorphic DNA (termed RAPD or arbitrarily primed PCR) by a single primer that does not have any known homology to the target sequence $[10,16]$. In the recent past, ribotyping performed with different restriction endonucleases (Sma I, Eco RI, BglI, Sal I, Acc I, HincII, Pst I and $S p h$ I) has been evaluated and a 'two-way ribotyping' procedure, with $P s t \mathrm{I}$ and $S p h \mathrm{I}$, has been found to be the best method for epidemiological purposes [6].

The aim of the present work was to develop a simpler and more rapid procedure than 'two-way ribotyping' to differentiate serotype Enteritidis strains. For this, PCR ribotyping and RAPD typing with three primers described as useful for the discrimination of Enteritidis strains $[10,16]$ as well as a ribotyping procedure with a mixture of Pst $\mathrm{I}$ and Sph I, termed 'PS ribotyping', were evaluated with a series that included strains associated with human infections and reference strains representing nine phage types. A dendrogram of genetic relationships between the PS ribotypes generated with the mixture of Pst I and Sph I was traced, and the resulting groupings correlated with RAPD types and phage types.

\section{Materials and methods}

\section{Bacterial strains}

This study included 65 strains of serotype Enteritidis associated with human infections, 49 of which were collected in different localities of the Principality of Asturias and 16 from other Spanish regions (Table 1). The selection of strains was based on their differences in some of the following parameters: isolation date, location or place where the salmonellosis episodes were registered, original sample and clinical manifestation. In these strains an epidemiological correlation was not apparent; 15 of the strains were associated with outbreaks. In addition, 11 Enteritidis reference strains were also tested. These were: ATTC 13076 ascribed to PT1; CECT 4155 ascribed to PT6a; type strains of PTs 4, 6, 6a, 8, 9 and 13a, from the Central Public Health Laboratory (CPHL), London; and type strains of PTs 1, 7 and 34 from Centro Nacional de Microbiología, Majadahonda, Madrid (CNM), Spain. Salmonella serotype Typhimurium ATCC 14028 was used to represent group B salmonellae. The pathogenic strains were phage typed in the Spanish Salmonella Reference Laboratory of the CNM by the scheme of Ward et al. [4].

\section{DNA isolation and ribotyping}

Genomic DNA isolation and ribotyping were done as described previously $[5,17]$. In the 'PS ribotyping', samples of $2 \mu \mathrm{g}$ of DNA were digested with $3 \mathrm{U}$ of Pst I and $5 \mathrm{U}$ of Sph I (Amersham) for $4 \mathrm{~h}$. DNA from isolates representing the different banding patterns (ribotypes) were tested three or more times to evaluate the reproducibility of the method. The ribotypes were named by the first letter of the restriction endonucleases used, which was followed by an arabic number corresponding to the detection order in this laboratory. In this study, only PS ribotypes from clinical and reference strains are shown, therefore some numbers of PS ribotypes are not included (they belonged to strains collected from other sources: foods, sewage, the environment).

\section{PCR-based procedures}

The DNA was obtained by the same procedure used for ribotyping. In PCR ribotyping the procedure and primers used were as described previously $[15,18]$. In RAPD typing three primers were used: MK22 [16], OPB6 and OPB17 [10]; the latter two were selected for their good discriminatory power. RAPD typing was performed with the conditions described previously for each primer $[10,16]$. DNA from isolates representing the different electrophoretic band patterns was tested three or more times to evaluate the reproducibility of the method. Minor differences in band intensity, as well as the weak bands, were not considered to define the PCR ribotypes and RAPD types. Different isolates were ascribed to the same PCR ribotype, or RAPD type, when their DNA amplified band patterns were considered to be identical. It is noteworthy that changes in amplification conditions produced different DNA amplified band patterns.

\section{Statistical analysis methods}

The discrimination index (DI), i.e., the probability that two unrelated strains sampled from the population would be placed into different typing groups, was calculated by Simpson's index of diversity [19]. For the similarity analysis of the Enteritidis ribotypes generated with the mixture of Pst I and SphI (also using Typhimurium ATCC 14028 to represent another salmonella group) the Dice similarity coefficient (S) was used. Cluster analysis was by an unweighted pair group method with arithmetic averages in the software programme MVSP (Multivariate Statistics Package, version $2.0 \mathrm{a})$. 


\section{Results}

\section{PCR based procedures}

Enteritidis strains representing nine phage types, lytic patterns that did not correspond to any recognised phage type (RDNC) and phage untypable (UPT) strains of the Ward et al. scheme, as well as 22 Pst I-Sph I combined ribotypes [6, and unpublished data] were tested by PCR ribotyping and RAPD typing. PCR ribotyping was not useful for the differentiation of strains of Enteritidis, because all the strains tested showed a single DNA amplified banding profile or PCR ribotype, which included three well-defined DNAamplified fragments ranging from 700 to $1000 \mathrm{bp}$ (figure not shown). RAPD typing was performed with three different primers: with primer MK22, all Enteritidis strains showed the same band profile or RAPD type, whereas with the primers OPB6 and OPB17 the series was differentiated into two and three RAPD types, respectively, and with each of these a similar but not identical distribution of strains into RAPD types was found (Fig. 1). With both primers, one RAPD type was found to be the -most frequent (labelled OPB6-1 and OPB17-1), which included all except two or five strains, respectively. The exceptions were: ATCC 13076 (PT1) and the type strain of PT8 yielded another RAPD type with each primer (labelled OPB6-2 and OPB17-2); the type strain of PT13a showed a combined RAPD type (OPB6-1, OPB17-2) and the two PT11 pathogenic strains gave OPB6-1 and a unique and specific OPB17 pattern labelled OPB17-3. With the three primers, the typability was $100 \%$, but the reproducibility was not good. In fact the banding patterns frequently included one or more DNAamplified fragments which were poorly defined, or not revealed in all the experiments. These fragments were considered artefactual bands and were not taken into account to define RAPD types. The number of RAPD types was increased to four by combining the results from OPB6 and OPB17. Moreover, by means of this combination, in the series tested (formed by 31 strains) the DI was very low, with a value of 0.31 .

\section{Ribotyping procedures}

In a first step, the same series of Enteritidis strains analysed by PCR-based methods and Typhimurium ATTC 14028 were tested by ribotyping performed with different mixtures of restriction endonucleases. The best differentiation, and the most accurate banding patterns, were obtained with the mixture of Pst I and $S p h$ I (this 'one-way' procedure was termed 'PS ribotyping'). The results of this mixture showed that strains ascribed to different phage types or combined-ribotypes, or both, generated different and well-differentiated profiles, labelled 'PS ribotypes', with the exception of type strains of PTs 4, 6 and 34 which gave the PS1 ribotype (Table 1, Fig. 2). In a second step, the other Enteritidis strains of the series were analysed by PS ribotyping. A total of 22 PS ribotypes was obtained, which included 7-12 fragments of sizes $27.5-2.3 \mathrm{~kb}$ (Fig. 2). No fragment was considered as common in all the PS ribotypes and the differences between pairs of ribotypes were easy to detect visually. Only a fragment of c. $7.5 \mathrm{~kb}$ (shown in some PS ribotypes displayed in Fig. 2, such as those labelled 2, $3,5,6,8,9,11,13,14$ and 17 , in which it is placed under a 7.8-kb fragment forming a double band) was not reproducible in all band patterns from the same strain. In some band patterns it appeared to be well defined, in others weak, and in others it was absent; and so it was not considered in the definition of PS ribotypes.

The distribution of strains into PS ribotypes is shown in Table 1 where it can be observed that nine PS ribotypes grouped two or more strains; the other 13 were represented by only one strain. Type strains of
MK22

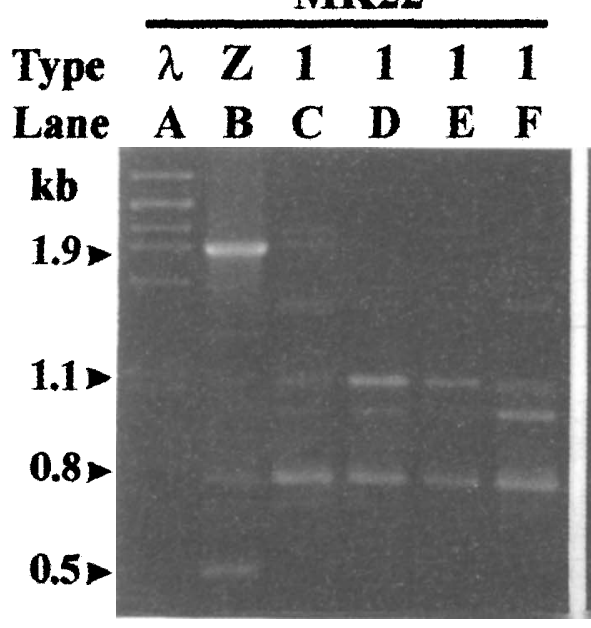

OPB6

\begin{tabular}{llllll}
\hline$\lambda$ & $\mathbf{Z}$ & $\mathbf{2}$ & $\mathbf{1}$ & $\mathbf{1}$ & $\mathbf{1}$ \\
$\mathbf{A}$ & $\mathbf{B}$ & $\mathbf{C}$ & $\mathbf{D}$ & $\mathbf{E}$ & $\mathbf{F}$
\end{tabular}

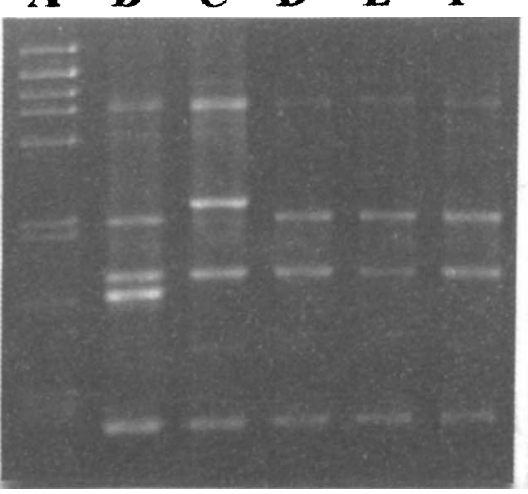

OPB17

Fig. 1. Analysis of genomic DNA of Salmonella strains by RAPD typing with the primers MK22, OPB6 and OPB17. Lanes A, DNA from $\lambda$ phage digested with $P_{s t} \mathrm{I}$; B, Typhimurium ATCC 14028; C-F, Enteritidis strains representing the four different combined RAPD types: C, type strain of PT8; D, type strain of PT4; E, 51/84 (a clinical strain ascribed to PT11); F, type strain of PT13a. The distribution of strains into RAPD types is shown in Table 1. 
Table 1. Typing of Salmonella serotype Enteritidis strains used in this study

\begin{tabular}{|c|c|c|c|c|c|c|c|c|c|c|c|c|c|}
\hline \multirow{2}{*}{$\begin{array}{l}\text { Strain } \\
\text { (ref./year) }\end{array}$} & \multicolumn{3}{|c|}{ Ribotype } & \multicolumn{2}{|c|}{ RAPD type } & \multirow{2}{*}{$\begin{array}{l}\text { Phage } \\
\text { type }\end{array}$} & \multirow{2}{*}{$\begin{array}{c}\text { Strain } \\
\text { (ref./year) }\end{array}$} & \multicolumn{3}{|c|}{ Ribotype } & \multicolumn{2}{|c|}{ RAPD type } & \multirow{2}{*}{$\begin{array}{l}\text { Phage } \\
\text { type }\end{array}$} \\
\hline & PS & $P$ & $\mathrm{~S}$ & OPB6 & OPB17 & & & PS & $\mathbf{P}$ & $S$ & OPB6 & OPB 17 & \\
\hline $117 / 87^{*}$ & 1 & 1 & 1 & 1 & 1 & PT 1 & $319 / 96^{*}$ & 3 & 1 & 23 & - & - & PT 1 \\
\hline $9693 / 94$ & 1 & 1 & 1 & - & - & PT 1 & $633 / 91^{*}$ & 3 & 1 & 23 & - & - & PT 4 \\
\hline $85 / 84$ & 1 & 1 & 1 & - & - & PT 4 & $424 / 93$ & 3 & 2 & 23 & 1 & 1 & PT 4 \\
\hline $801 / 91$ & 1 & 1 & 1 & - & - & PT 4 & $474 / 93$ & 3 & 2 & 23 & - & - & PT 4 \\
\hline $275 / 93$ & 1 & 1 & 1 & - & - & PT 4 & $7452 / 94$ & 3 & 2 & 23 & - & - & RDNC \\
\hline $305 / 93$ & 1 & 1 & 1 & - & - & PT 4 & $276 / 93^{*}$ & 5 & 1 & 12 & 1 & 1 & PT 4 \\
\hline $316 / 93$ & 1 & 1 & 1 & - & - & PT 4 & $354 / 93$ & 5 & 1 & 12 & - & - & PT 4 \\
\hline $385 / 93$ & 1 & 1 & $i$ & - & - & PT 4 & $418 / 93$ & 5 & 1 & 12 & - & - & PT 4 \\
\hline $393 / 93$ & 1 & 1 & 1 & - & - & PT 4 & $44 / 84$ & 6 & 1 & 5 & 1 & 1 & $\mathrm{PT} 4$ \\
\hline $398 / 93^{*}$ & 1 & 1 & 1 & - & - & PT 4 & $45 / 93$ & 6 & 2 & 5 & 1 & 1 & PT 4 \\
\hline $87 / 94$ & 1 & 1 & 1 & - & - & PT 4 & $51 / 84$ & 7 & 8 & 2 & 1 & 3 & PT 11 \\
\hline $233 / 94$ & 1 & 1 & 1 & - & - & PT 4 & $138 / 87$ & 7 & 8 & 2 & 1 & 3 & PT 11 \\
\hline $8328 / 94$ & 1 & 1 & 1 & - & - & PT 4 & $124 / 85^{*}$ & 8 & 2 & 8 & 1 & 1 & PT $6 a$ \\
\hline $9221 / 94$ & 1 & 1 & 1 & - & - & PT 4 & $125 / 85^{*}$ & 8 & 2 & 8 & - & - & RDNC \\
\hline $195 / 93$ & 1 & 1 & 1 & - & - & PT $5 \mathrm{a}$ & $297 / 93$ & 9 & 1 & 9 & - & - & PT 4 \\
\hline $78881 \dagger$ & 1 & 1 & 1 & - & - & PT 6 & $98 / 87$ & 9 & 1 & 9 & 1 & 1 & UPT \\
\hline $89468 \dagger$ & 1 & 1 & 1 & - & - & PT 6 & $408 / 93^{*}$ & 10 & 1 & 11 & 1 & 1 & PT 4 \\
\hline $632 / 91^{*}$ & 1 & 1 & 1 & - & - & PT 6 & $437 / 93$ & 10 & 1 & 11 & - & - & UPT \\
\hline $707 / 91$ & 1 & 1 & 1 & - & - & PT 6 & $20 / 84^{*}$ & 11 & 2 & 1 & 1 & 1 & PT 1 \\
\hline $44 / 93$ & 1 & 1 & 1 & - & - & PT 6 & $80 / 87^{*}$ & 12 & 7 & 7 & 1 & 1 & RDNC \\
\hline $197 / 93$ & 1 & 1 & 1 & - & - & PT 6 & $88 / 87^{*}$ & 13 & 1 & 1 & 1 & 1 & PT 4 \\
\hline $211 / 93$ & 1 & 1 & 1 & - & - & PT 6 & $15 / 90$ & 14 & 1 & 1 & 1 & 1 & PT 6 \\
\hline $1585 / 93$ & 1 & 1 & 1 & - & - & PT 6 & $5728 / 94$ & 15 & 1 & 15 & 1 & 1 & PT 8 \\
\hline $2550 / 94$ & 1 & 1 & 1 & - & - & PT 6 & $475 / 93$ & 16 & 5 & 1 & 1 & 1 & UPT \\
\hline $8805 / 94$ & 1 & 1 & 1 & - & - & PT 6 & $11 / 94$ & 17 & 1 & 13 & 1 & 1 & PT 1 \\
\hline $380 / 93$ & 1 & 1 & 1 & - & - & PT $6 a$ & $115 / 94$ & 18 & 4 & 4 & 1 & 1 & PT 4 \\
\hline $384 / 93$ & 1 & 1 & 1 & - & - & PT 6a & & & & & & & \\
\hline $3468 / 94$ & 1 & 1 & 1 & - & - & PT 6a & \multicolumn{2}{|c|}{ Reference strains } & & & & & \\
\hline $7458 / 94$ & 1 & 1 & 1 & - & - & PT 6a & CPHL & 1 & 1 & 1 & 1 & 1 & PT 4 \\
\hline $78 / 84$ & 1 & 1 & 1 & 1 & 1 & PT 7 & CPHL & 1 & 1 & 1 & 1 & 1 & PT 6 \\
\hline $355 / 94^{*}$ & 1 & 1 & 1 & - & - & PT 7 & CNM 5209 & 1 & 1 & 1 & 1 & 1 & PT 34 \\
\hline $9687 / 94$ & 1 & 1 & 1 & - & - & PT 34 & CNM 5011 & 3 & 1 & 23 & 1 & 1 & PT 1 \\
\hline $55 / 87^{*}$ & 1 & 1 & 1 & - & - & RDNC & ATCC 13076 & 3 & 1 & 23 & 2 & 2 & PT 1 \\
\hline $919 / 94$ & 1 & 1 & 1 & - & - & RDNC & CNM 4557 & 3 & 1 & 23 & 1 & 1 & PT 7 \\
\hline $4073 / 94$ & 1 & 1 & 1 & - & - & RDNC & CECT 4155 & 20 & 1 & 28 & 1 & 1 & PT 6a \\
\hline $217 / 93^{*}$ & 2 & 1 & 7 & 1 & 1 & PT 6a & CPHL & 21 & 1 & 25 & 2 & 2 & PT 8 \\
\hline $313 / 93$ & 2 & 1 & 7 & - & - & PT 6a & CPHL & 22 & 1 & 26 & 1 & 2 & PT $13 a$ \\
\hline $216 / 94$ & 2 & 1 & 7 & - & - & PT $6 \mathrm{a}$ & CPHL & 24 & 14 & 24 & 1 & 1 & PT 9 \\
\hline $299 / 94$ & 2 & 1 & 7 & - & - & PT 6a & CPHL & 30 & 15 & 27 & 1 & 1 & PT $6 \mathrm{a}$ \\
\hline
\end{tabular}

Strains are listed in order according to their ribotypes beginning with the mixture $P s t \mathbf{I}$ and $S p h \mathbf{I}$.

$\mathrm{RDNC}$, strains reacted with the typing phages but did not correspond to any recognised phage type; UPT, phage untypable by the scheme of Ward et al. [4].

Reference strains were from: ATCC, American Type Culture Collection; CPHL, Central Public Health Laboratory, London; CNM, Centro Nacional de Microbiología, Madrid; CECT, Colección Española de Cultivos Tipo.

${ }^{*}$ Strains associated with outbreaks. The number of isolates tested from each outbreak was $>1$.

†Isolation year not known, but earlier than 1987.

PTs 6a, 8, 9 and 13a (from CPHL) showed PS ribotypes that were not found among the Spanish strains tested.

The present series has been analysed in previous studies [6, unpublished data] by ribotyping performed separately with Pst I and Sph I or 'two-way ribotyping', and it was differentiated into eight ribotypes with Pst I, into 17 with SphI and into 22 by the combination of types from both enzymes. The capacity to differentiate Enteritidis strains by PS ribotyping and two-way ribotyping procedures was compared, and a similar but not identical distribution of strains into types was found (Table 1). The differences recorded were as follows. (i) All except two strains with P1-S1 ribotypes fell into the PS1 ribotype. One was ascribed to PS13 ribotype, which had three additional fragments of 13.1, 11.5 and $9.4 \mathrm{~kb}$ that were not present in PS1 ribotype (Fig. 2, lane C). The other strain was ascribed to the PS14 ribotype, which showed only an additional fragment of c. $6 \mathrm{~kb}$, with respect to PS1 ribotype (Fig. 2, lane D). (ii) All strains showing $\mathrm{P} 1-\mathrm{S} 23$ or $\mathrm{P} 2-\mathrm{S} 23$ ribotypes fell into the PS3 ribotype (Fig. 2, lanes $\mathrm{E}$ and F). (iii) The only two strains showing P1-S5 or P2-S5 ribotypes fell into the PS6 ribotype (Fig. 2, lanes I and $\mathrm{J}$ ).

On the other hand, the comparison of banding patterns generated by Pst I and Sph I used alone or in mixture allowed the number of Pst I-fragments that were cut by Sph I and vice versa to be deduced. The Pst I ribotypes included seven or less fragments $>7 \mathrm{~kb}$, suggesting that Pst I had not cut points into the rrn loci. The Sph I ribotypes showed 5-12 fragments of $3.5->27.5 \mathrm{~kb}$, supporting the fact that $S p h$ I cuts into some of the rrn loci [6]. As an example, the banding patterns of $\mathrm{P} 1, \mathrm{~S} 1$, and PS1 ribotypes are shown in 


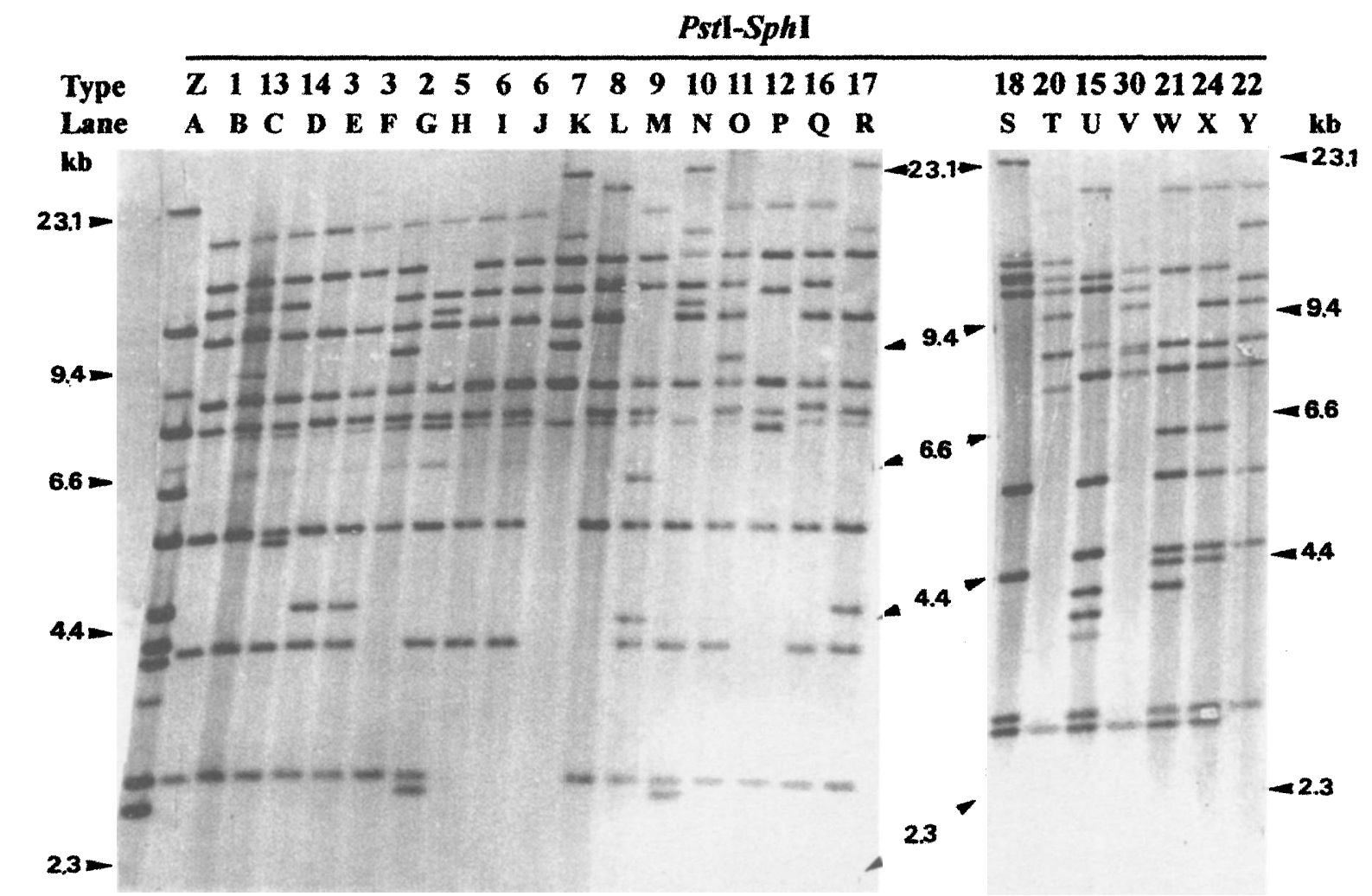

Fig. 2. Ribotyping performed with a mixture of Pst I and Sph I of Enteritidis strains. Lane A, PS ribotype of Typhimurium ATCC 14028, other lanes show PS ribotypes of representative Enteritidis strains: B, phage type strain of PT4; C, 88/87; D, 15/90; E, ATCC 13076; F, 424/93; G, 217/93; H, 276/93; I, 44/84; J, 45/93; K, 51/84; L, $124 / 85 ; \mathbf{M}, 297 / 93 ; \mathbf{N}, 408 / 93 ; \mathbf{O}, 20 / 84 ; \mathbf{P}, 80 / 87 ; \mathbf{Q}, 475 / 93 ; \mathbf{R}, 11 / 94 ; \mathbf{S}, 115 / 94 ; \mathbf{T}$, CECT 4155; U, 5728; V, type strain of PT6a; W, type strain of PT8; X, type strain of PT9; Y, type strain of PT13a. The distribution of strains into PS ribotypes is shown in Table 1.

Fig. 3. Comparison of patterns reveals that in the PS1 ribotype, four operons (carried by Pst I fragments of $15.1,12.9,10.7$ and $8.5 \mathrm{~kb}$, indicated by arrows in Fig. 3) have no $S p h$ I cut points. It also revealed that in the sequences adjacent to the $r r n$ loci, the $S p h$ I cut points are placed further from the rrn loci than Pst I cut points. For this reason, when a mixture of both enzymes was used, the largest Sph I fragments $(>27 \mathrm{~kb})$ were missing and were not revealed in the PS ribotypes. On the other hand, the largest Sph I fragments frequently appeared to be poorly differentiated (as can be observed in the figures of references [6 and 20]) while all the fragments forming the PS ribotypes were visually well-differentiated.

In both ribotyping procedures, reproducibility (with the DNA well cleaved) and typability reached $100 \%$, and in the series tested here, the discrimination index was a little higher with PS ribotyping than with twoway ribotyping, reaching values of $\mathrm{DI}=0.74$ and $\mathrm{DI}=0.72$, respectively. Three important features, the improved 'quality' of the banding patterns, the lower cost in time and work, and the slightly higher DI of PS ribotyping compared to two-way ribotyping, led us to support the first procedure, and its usefulness for phylogenetic and epidemiological purposes is commented on in the following section of this study.
Dendrogram of genetic relationship among $P S$ ribotypes: correlation among $P S$ ribotypes, $R A P D$ types and phage types

The sequence divergence of the rDNA regions (which include the $r r n$ loci and adjacent sequences) of the strains under study can be revealed by comparison of the fragment restriction length polymorphisms that defined their ribotypes. With data from the 22 PS ribotypes found within Enteritidis strains and the PS ribotype of TS, Typhimurium ATCC 14028, used as an outgroup strain, a dendrogram of genetic similarity was traced (Fig. 4). It showed a high degree of heterogeneity in the sequences of the rDNA regions of Enteritidis, because their similarity coefficients were in the range $0.95-0.35$ (similarity 95-35\%). At the lowest significance level, $\mathrm{S}=0.35$, all Enteritidis ribotypes were grouped whereas Typhimurium ATCC 14028 was situated out of the group. Moreover, at a significance level of 0.7 , a major cluster (including 12 PS ribotypes, $84.2 \%$ of strains) was revealed and was labelled cluster A.

The grouping arising from the dendrogram was correlated with phage types and RAPD types, revealing the following factors. (i) All strains ascribed to PTs 1, 5a, 6, 7 and 34, together with all except two PT 4 and 6a strains, and all except one RDNC and one UPT strain, fall into cluster A. All these strains showed the prevalent RAPD 

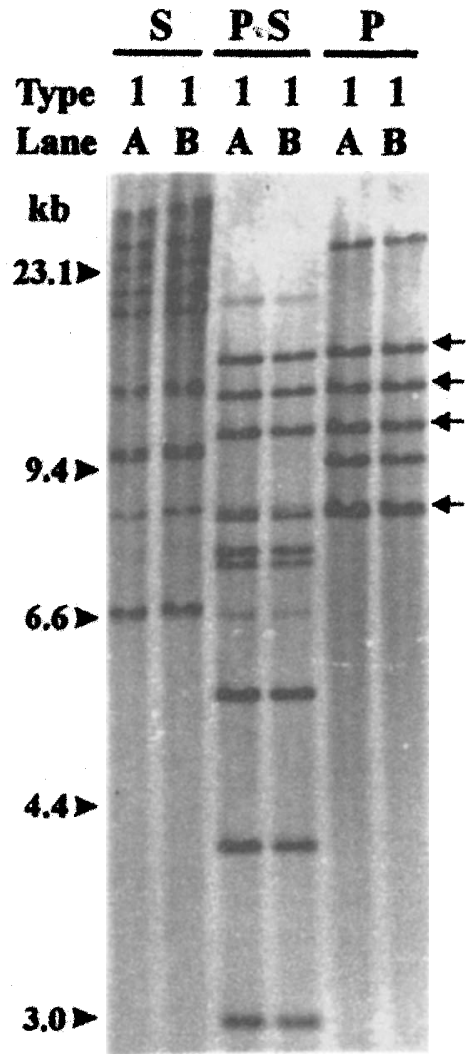

Fig. 3. Ribotyping of Enteritidis strains performed with Pst I and Sph I alone or in combination. Lanes $\mathbf{A}, 85 / 84$ - an Asturian clinical strain ascribed to PT4; B, 117/87 - an Asturian clinical strain ascribed to PT1. S, banding patterns from ribotyping performed with $S p h \mathrm{I}$, representing S1 ribotype; PS, banding patterns from ribotyping performed with a mixture of $S p h \mathrm{I}$ and Pst I, representing PS1 ribotype; $\mathbf{P}$, banding patterns from ribotyping performed with Pst $\mathrm{I}$, representing $\mathrm{P} 1$ ribotype. Arrows show the four Pst I fragments not cut by Sph I.

types (OPB6-1 and OPB17-1), except the reference strain ATCC 13076 (PT1) which showed OPB6-2 and OPB17. 2. (ii) The only two PT6a strains not included in cluster A showed the prevalent RAPD types, but their PS ribotypes were the most loosely related to the remaining branches of the dendrogram. These strains were the type strain of PT6a and CECT 4155 showing PS30 and PS20 ribotypes, respectively, with $76 \%$ similarity between them. (iii) The strains belonging to PT8 and PT9 fell into a minor cluster, at a 0.65 similarity level, labelled cluster B, which included three ribotypes, each of these represented by only one strain: PS21 by the type strain of PT8 (OPB62 and OPB 17-2), PS23 by the type strain of PT9 (OPB61, OPB17-1) and PS15 by the single Spanish clinical strain ascribed to PT8 (OPB6-1, OPB17-1). (iv) The PS7 ribotype represented by the only two strains ascribed to PT11 (OPB6-1, OPB17-3) showed $<52 \%$ similarity with respect to the remaining Enteritidis ribotypes.

\section{Discussion}

Several criteria should be considered in evaluating genetic typing procedures for epidemiological pur- poses [21]. In all the procedures used in the present work, the stability of the marker screening and the typability reached $100 \%$. The reproducibility could be considered very good in ribotyping, but poor in PCRbased methods, because their banding patterns frequently included fragments that were not well resolved. The sensitivity or discriminatory power by the calculation of a DI (which takes into consideration both the number of types defined and their relative frequencies) was zero with PCR ribotyping; 0-0.31 with RAPD procedures; and 0.74 with PS ribotyping. The latter is among the highest values reported for a typing method for human pathogenic Enteritidis strains. It is noteworthy that within PT4, PS ribotyping differentiated eight PS ribotypes and reached a $\mathrm{DI}=0.72$, which is also among the highest values reported for this phage type. The easy interpretation of band patterns and the ease of comparison of the results from different experiments was better in ribotyping than in RAPD typing. An additional observation is underlined: the band patterns were better differentiated by the one-way or PS than by the two-way ribotyping. This was due to the fact that with Sph I alone, the interpretation of the number and size of the fragments placed in the upper region $(>20 \mathrm{~kb}$, which differentiate several types) can be difficult to visualise (as can be seen in Fig. 3, tracks S, and references [6 and 20]). Whereas when $S p h$ I was used in mixture (or on previously digested DNA) with Pst I the problems in the digestions and interpretation of the new banding patterns did not arise, as the largest $S p h$ I fragments were cleaved by $P s t \mathrm{I}$.

The results of this series with PCR-based typing methods were compared with results described previously, in which the same primers were used. Lagatolla et al. [18] analysed a panel of 218 strains of Salmonella (belonging to 10 serovars and isolated in Italian hospitals during 1977-1994) by PCR ribotyping. The 41 Enteritidis isolates showed a single identical banding profile, consisting of four DNA amplified fragments of $1100-700 \mathrm{bp}$, which was different from the profiles arising from other serotypes. It was also different from the single PCR ribotype found in the present Enteritidis series, which includes only three well-defined fragments in the region $1000-700 \mathrm{bp}$. The first RAPD analysis applied to Enteritidis was developed by Fadl et al. [16], and used primer MK22 to analyse strains from 12 human outbreaks and from 20 avian sources (belonging to PT8, PT13a, PT14b and UPT). Seven distinct randomly amplified DNA patterns were found; four patterns were represented by strains from avian and human sources. In the present series, represented by 10 different phage types (which did not include PTs 13 or $14 b$ and only the phage type strain of PT13a) no differentiation of strains was revealed. Moreover, the band patterns from both series showed DNA amplified fragments in the region $2200-700 \mathrm{bp}$, and two fragments (of 700 and $1150 \mathrm{bp}$ ) seemed to be common 


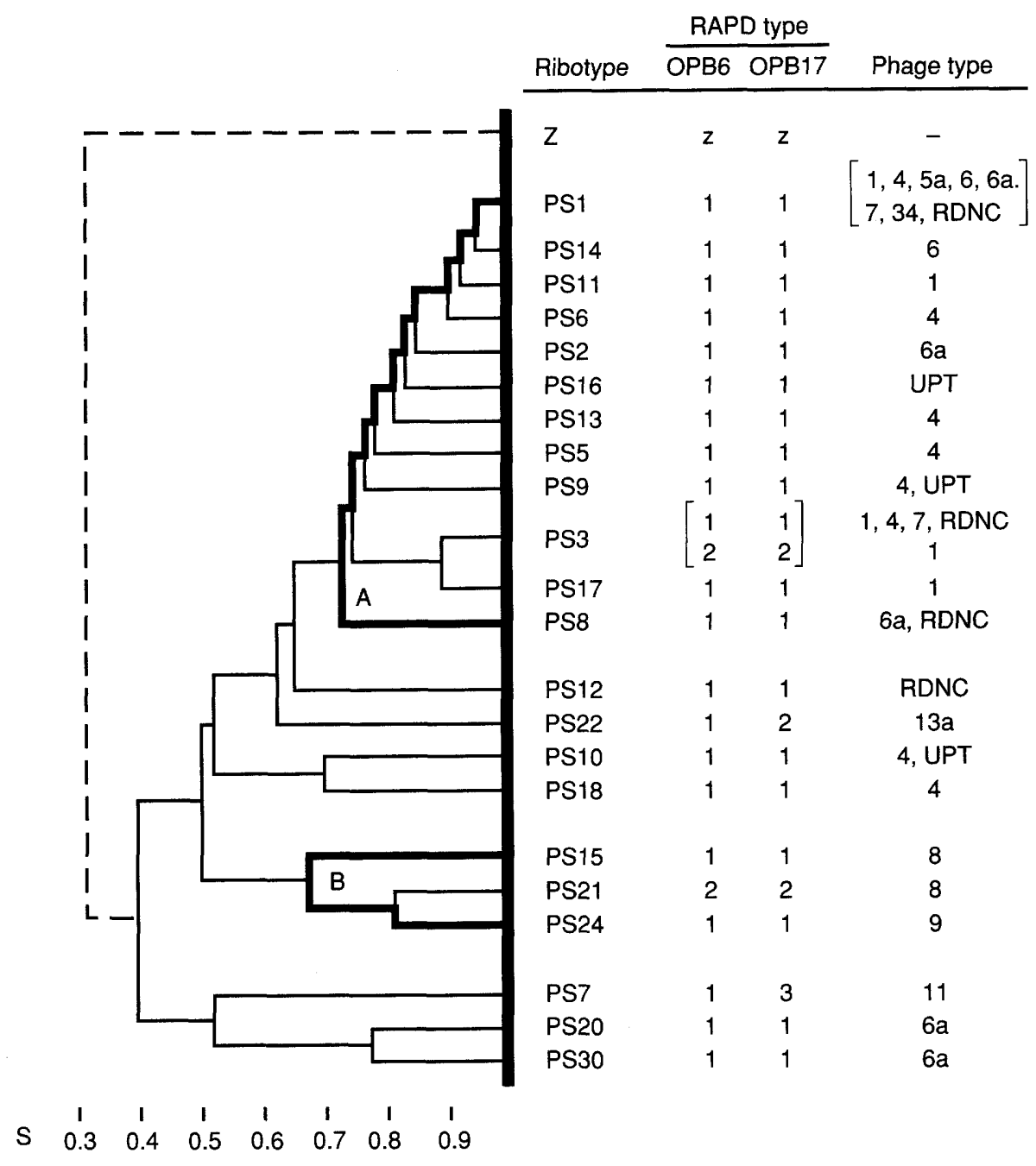

Fig. 4. Single linkage dendrogram showing the results of the cluster analysis on the basis of the PS ribotypes of the Enteritidis strains. Correlation with RAPD types and phage types. Clusters A and $\mathbf{B}$ are framed by dark lines. PS ribotype of Typhimurium ATCC 14028 is labelled Z and indicated by the dotted line. S, similarity coefficient.

in all the band patterns. In another recent work, Lin et al. [10] selected six arbitrary primers (from the 65 primers screened) which were used to test 28 Enteritidis isolates, collected in various USA states and one in Germany. Different primers produced different numbers of patterns of RAPD types, ranging from three to five. When we evaluated the primers called OPB6 and OPB17 [10], our series was differentiated into two and three patterns, respectively. The most frequent RAPD types in our series were not found in the USA series; but RAPD types labelled OPB6-2 and OPB17-2 in our series - shown only by reference strain ATCC 13076 (PT1) and the type strains of PT8 and PT13a - seem to be identical to OPB6-A and OPB17-C, respectively, both frequent in the USA series.

The development of a new typing method must include an adequate understanding of its effectiveness for a broad sample of strains, including wellcharacterised reference strains, epidemic and sporadic isolates; and the results should be compared directly with those of previous well-studied approaches. PS ribotyping was evaluated on a series meeting these requirements, and the results were compared with phage typing, two-way ribotyping and PCR-based typing, which are methods described as useful in Enteritidis typing $[4,6,10,16,18]$. The data described above support PS ribotyping as the most accurate and discriminative of the typing procedures tested in the present study. Some observations derived from the application of the combination of PS ribotyping with phage typing, as an epidemiological tool, can be considered interesting. (i) The presence of a most frequent type or clonal line (PT4, PS1 ribotype) among Spanish pathogenic strains producing different clinical manifestations (acute enteritis, septicaemia and focal infections), which was also represented by strains collected from egg dishes, chicken and meats categorised as infection vehicles (data not shown). It showed a broad dispersion in space and time, because PT4-PS1 strains were collected in different geographic areas situated in the Iberian Peninsula and Canary Isles from 1984 to 1995. It also includes the type strain PT4 from England. (ii) This, and other frequent types - PT6-PS1, PT6a-PS1, PT6a-PS2, PT4-PS3 and 
PT4-PS5 - fall into a major cluster in the dendrogram, and can be considered as members of a prevalent lineage. It has been reported that strains of these PTs, which are the most frequent in Europe, showed an identical IS200 profile, that is different from the IS200 profile shown by PTs, 8, 13, 13a, and 14 which are the most frequent in the USA $[2,3,10,13]$. (iii) With data from IS 200 patterns, the PT11 pathogenic strains have been considered to be members of another lineage $[8,13]$; this is also supported by data from the present work, where PT11 strains were ascribed to PS7 ribotype, which appears as a branch loosely related with the major cluster in the dendrogram. The two PT11 strains also showed a specific and different RAPD type with the OPB17 arbitrary primer. (iv) Human pathogenic strains representing PTs 1, 4, 6, 6a, 7, 8, RDNC and UPT were differentiated by ribotyping. Conversely, strains showing PS1 ribotype were differentiated into several phage types. The data support PS ribotyping as a complementary or alternative typing method to phage typing for serotype Enteritidis.

Finally, in a routine typing method features such as ease of operation, rapidity and cost are also important. The great advantage of PCR-based methods is that these are faster, relatively simpler and more economical than other genomic typing methods. Therefore, RAPD procedures that analyse the entire bacterial chromosome emerge as particularly attractive molecular typing methods, but their application requires the identification of the oligonucleotide primers that are capable of recognising DNA polymorphisms among isolates and, unfortunately, there is no way of predicting which oligonucleotide sequences will be useful to differentiate strains of a species or serotype. At the present time, more work is necessary to develop a well-standardised RAPD typing procedure, with a low number of selected primers that yield specific and well-defined DNA amplified fragments to be able to compare results from different series and laboratories. On the contrary, ribotyping is a laborious procedure, as multiple steps are required (DNA isolation, restriction, electrophoresis, Southern blotting, probe preparation and hybridisation); the proposal of performing it with a single restriction-hybridisation step is the most simple approach. Despite this disadvantage, currently, we support the ribotyping procedure performed with the mixture of Pst I and $S p h \mathrm{I}$ and the $r r n B$ operon (or 16S and 23S rRNAs) as probe, as the most useful tool to differentiate Enteritidis pathogenic strains. The use of PS ribotyping is re-inforced by three important observations: (i) its high degree of typability, stability and reproducibility, (ii) its good sensitivity to differentiate strains of several phage types, and (iii) the ease of interpretation of the ribotypes, that include distinct and wellresolved fragments which will guarantee the accurate comparison of results obtained from different series in different laboratories.
We thank Dr A. Aladueña, Dr M. A. Usera and CNM for the phage typing of the Enteritidis strains and reference strains; $\mathrm{Dr} \mathrm{M}$. A. González-Hevia and CNM for pathogenic Enteritidis isolates; and Dr M. Altwegg for pKK3535 plasmid. E. Landeras was the recipient of a grant from Oviedo University. This work was supported by a grant from Fondo de Investigación Sanitaria Español (Ref. 95/0030-01).

\section{References}

1. Rodrigue DC, Tauxe RV, Rowe B. International increase in Salmonella enteritidis: a new pandemic? Epidemiol Infect 1990; 105: 21-27.

2. Stanley J, Burnens AP, Threlfall EJ, Chowdry N, Goldsworthy M. Genetic relationships among strains of Salmonella enteritidis in a national epidemic in Switzerland. Epidemiol Infect 1992; 108: 213-220.

3. Usera MA, Popovic T, Bopp CA, Strockbine NA. Molecular subtyping of Salmonella enteritidis phage type 8 strains from the United States. J Clin Microbiol 1994; 32: 194-198.

4. Ward LR, De Sa JDH, Rowe B. A phage-typing scheme for Salmonella enteritidis. Epidemiol Infect 1987; 99: 291-294.

5. González-Hevia MA, Mendoza MC. Polymorphism of rRNA genes and plasmid analysis in the typing of Salmonella enterica serovar Enteritidis from a Spanish health area. New Microbiol 1995; 18: 377-384.

6. Landeras E, González-Hevia MA, Alzugaray R, Mendoza MC. Epidemiological differentiation of pathogenic strains of Salmonella enteritidis by ribotyping. J Clin Microbiol 1996, 34: 2294-2296.

7. Rampling A. Salmonella enteritidis five years on. Lancet 1993; 342: $317-318$.

8. Olsen JE, Skov MN, Threlfall EJ, Brown DJ. Clonal lines of Salmonella enterica serotype Enteritidis documented by IS200-, ribo-, pulsed-field gel electrophoresis and RFLP typing. $J$ Med Microbiol 1994; 40: 15-22.

9. D'Aoust JY. Salmonella and the international food trade. Int $J$ Food Microbiol 1994; 24: 11-31.

10. Lin AW, Usera MA, Barrett TJ, Goldsby RA. Application of random amplified polymorphic DNA analysis to differentiate strains of Salmonella enteritidis. J Clin Microbiol 1996; 34: $870-876$.

11. Powell NG, Threlfall EJ, Chart H, Rowe B. Subdivision of Salmonella enteritidis PT4 by pulsed-field electrophoresis: potential for epidemiological surveillance. FEMS Microbiol Lett 1994; 119: 193-198.

12. González-Hevia MA, Mendoza MC. Differentiation of strains from a food-borne outbreak of Salmonella enterica by phenotypic and genetic typing methods. Eur $J$ Epidemiol 1995; 11: 479-482.

13. Stanley J, Goldsworthy M, Threlfall EJ. Molecular phylogenetic typing of pandemic isolates of Salmonella enteritidis. FEMS Microbiol Lett 1992; 90: 153-160.

14. Helmuth R, Schroeter A. Molecular typing methods for $S$. enteritidis. Int J Food Microbiol 1994; 21: 69-77.

15. Kostman JR, Edlind TD, LiPuma JJ, Stull TL. Molecular epidemiology of Pseudomonas cepacia determined by polymerase chain reaction ribotyping. $J$ Clin Microbiol 1992; 30: 2084-2087.

16. Fadl AA, Nguyen AV, Khan MI. Analysis of Salmonella enteritidis isolates by arbitrarily primed PCR. J Clin Microbiol 1995; 33: 987-989.

17. Mendoza MC, Alzugaray R, Landeras E, González-Hevia MA. Discriminatory power and application of ribotyping of Yersinia enterocolitica 0:3 in an epidemiological study. Eur J Clin Microbiol Infect Dis 1996; 15: 220-226.

18. Lagatolla C, Dolzani L, Tonin E et al. PCR ribotyping for characterizing Salmonella isolates of different serotypes. $J$ Clin Microbiol 1996; 34: 2440-2443.

19. Hunter PR, Gaston MA. Numerical index of the discriminatory ability of typing systems: an application of Simpson' index of diversity. J Clin Microbiol 1988; 26: 2465-2466.

20. Gruner E, Martinetti Lucchini G, Hoop RK, Altwegg $M$. Molecular epidemiology of Salmonella enteritidis. Eur $J$ Epidemiol 1994; 10: 85-89.

21. Maslow JN, Mulligan ME, Arbeit RD. Molecular epidemiology: application of contemporary techniques to the typing of microogransims. Clin Infect Dis 1993; 17: 153-164. 